\title{
artigo de atualização Qualidade de vida e TDAH
}

\section{Quality of life and $A D H D$}

\author{
Paulo Mattos', Gabriel Coutinho²
}

\section{RESUMO}

Portadores adultos de transtorno de déficit de atenção/hiperatividade (TDAH) apresentam comprometimento funcional em diferentes áreas da vida diária que deve ser investigado durante a avaliação clínica. Apesar disso, até recentemente não existiam instrumentos para avaliar o impacto do TDAH na qualidade de vida de adultos portadores. O Adult ADHD Quality of Life Questionnaire (AAQoL) é um instrumento desenhado a partir da sistematização de um conjunto de informações na literatura especializada sobre impacto da doença e desfechos clínicos, bem como opinião de especialistas, de modo a avaliar quantitativamen-

\section{Palavras-chaves}

Transtorno de déficit de atenção/hiperatividade, adultos, qualidade de vida, AAQOL.

\section{Key-words}

Attention deficit hyperactivity disorder, adults, quality of life, AAQoL. te a qualidade de vida de portadores adultos. O instrumento é subdividido em quatro diferentes subescalas, denominadas produtividade, saúde psicológica, perspectivas de vida e relacionamentos. É discutida a utilidade de um questionário especificamente desenvolvido para avaliar qualidade de vida no TDAH adulto e é apresentado o projeto de validação semântica, validade de constructo e confiabilidade do instrumento em andamento.

\section{ABSTRACT}

Adults with attention deficit hyperactivity disorder (ADHD) present higher rates of functional impairment in several areas of daily life which must be investigated during clinical evaluation. Nevertheless, until recently there were no instruments available to measure the impact of ADHD in adult patient's quality of life. The Adult ADHD Quality of Life Questionnaire (AAQoL) was developed based on literature reports of the burden of ADHD and its outcomes as well as experts' reports in order to quantify quality of life levels. The instrument consists of four different domains: life productivity, psychological healthy, relationships and life outlook. The present paper will discuss the usefulness of a questionnaire specially developed to measure adult ADHD quality of life and also portray the project of semantic validation; construct validity and reliability of the instrument under progress.

GEDA - Grupo de Estudos do Déficit de Atenção do Instituto de Psiquiatria da UFRJ.

1 Universidade Federal do Rio de Janeiro (UFRJ).

2 Instituto de Psiquiatria da Universidade Federal do Rio de Janeiro (IPUB-UFRJ). 


\section{INTRODUÇÃO}

Estudos longitudinais demonstraram que o transtorno de déficit de atenção/hiperatividade (TDAH) persiste na vida adulta em torno de $60 \%$ a $70 \%$ dos casos (Barkley et al., 2002), sendo as diferenças encontradas nas taxas de remissão provavelmente secundárias às diferentes definições de TDAH ao longo do tempo e aos critérios para o seu diagnóstico (Biederman et al., 2000). O diagnóstico de TDAH em adultos é realizado, de modo ideal, utilizando-se critérios bem definidos, tais como os empregados pelo sistema DSM. Os sintomas listados no DSM-IV para o diagnóstico de crianças e adolescentes foram adaptados para adultos na escala auto-aplicada ASRS - Escala de Auto-Relato do TDAH em Adultos (Adult Self-Report Scale, versão 1.1) (Kessler et al., 2005) validada recentemente no nosso meio (Mattos et al., 2006). Embora a escala não permita o diagnóstico per se, ela é útil no rastreio e de grande benefício na sugestão de aspectos específicos a serem discutidos com o paciente durante a consulta. Para um diagnóstico clínico tal como exigido em ambiente de pesquisa, emprega-se o módulo de TDAH do questionário K-SADS-E adaptado para adultos (Grevet et al., 2005). Cumpre ressaltar que esse questionário, mesmo para uso em crianças, na sua versão original em inglês, possui alguns problemas (Mattos e Rohde, in press).

Num estudo epidemiológico recente (Kooij et al., 2005), a validade do diagnóstico de TDAH em adultos foi demonstrada por meio da análise fatorial de sintomas auto-relatados numa população adulta, na qual aqueles indivíduos com maior número de sintomas nucleares de TDAH apresentavam piores indicadores de funcionamento global. Outro recente estudo epidemiológico também demonstrou que adultos com diagnóstico de TDAH tendiam a apresentar piores escores em escalas de avaliação de funcionamento global e comprometimento numa série de medidas cognitivas (Kessler et al., 2006).

\section{Instrumentos para avaliação de qualidade de vida}

Muito embora diferentes evidências apontem para um grande comprometimento funcional no cotidiano dos portadores adultos com o transtorno, não havia, até recentemente, um instrumento de avaliação específico de qualidade de vida no TDAH. Uma alternativa seria o questionário SF-36 (Ware et al., 2000), validado e reconhecido para avaliar qualidade de vida geral. O instrumento disponibiliza escores em oito subescalas: capacidade funcional, aspecto físico, dor, estado geral da saúde, vitalidade, aspecto social, aspecto emocional, saúde mental. Essa escala foi utilizada em estudo com amostra de adultos portadores de TDAH (Adler et al., 2006), e os achados demonstraram que apenas quatro das subescalas (vitalidade, aspecto social, aspecto emocional e saúde mental) melho- ravam com a remissão de sintomas de TDAH, ao passo que as outras quatro subescalas (aspecto físico, dor, estado da saúde geral e capacidade funcional) não se correlacionavam com a melhora nos sintomas de TDAH. Outro estudo que utilizou o SF-36 como medida de qualidade de vida em portadores de TDAH (Landgraf, in press) também utilizou apenas as mesmas quatro subescalas, tendo em vista que as outras não apresentavam correlação com o impacto dos sintomas de TDAH na qualidade de vida do portador.

\section{Questionário de Qualidade de Vida em Adultos com TDAH (AAQoL)}

Para preencher a lacuna de um instrumento específico para avaliar qualidade de vida em adultos portadores de TDAH, foi desenvolvido o Adult ADHD Quality of Life Questionnaire (AAQoL) ou Questionário de Qualidade de Vida em Adultos com TDAH (Brod et al., 2006). A sua estrutura foi desenvolvida a partir da sistematização de um conjunto de informações sobre os sintomas e o impacto da doença, colhido com os próprios pacientes, com especialistas em TDAH e na literatura específica. Essas informações possibilitaram aos autores a criação de um modelo conceitual sobre o impacto do TDAH do adulto na qualidade de vida do indivíduo. Em sua versão atual o AAQoL é uma escala do tipo Likert constituída por 29 itens distribuídos em quatro subescalas denominadas: produtividade (11 itens), saúde psicológica (6 itens), perspectivas de vida (7 itens) e relacionamentos (5 itens).

No estudo original de validação da escala, a validade de constructo do AAQoL foi confirmada em relação ao conjunto total dos seus itens, assim como em relação às quatro dimensões (subescalas) do instrumento. A consistência interna da escala se mostrou substancial, obtendo-se um alfa de Cronbach's de 0,93. Com base nesses resultados, os autores concluíram que o AAQoL é um instrumento válido para avaliar a qualidade de vida em adultos com TDAH e que deveria ser incorporado em estudos sobre o transtorno.

No estudo de validação, verificou-se que os portadores de TDAH apresentavam maior número de divórcios, maiores taxas de desemprego e menor renda média se comparados aos não-portadores, embora ambos os grupos se equiparassem etnicamente e em anos de estudo. Esses achados vão ao encontro do modelo no qual o AAQoL foi construído, sugerindo comprometimento mais acentuado na produtividade e nos relacionamentos (duas das áreas avaliadas pelo instrumento) que se traduzem em discrepâncias clinicamente significativas em taxas de emprego, casamento e rendimento médio. Mais ainda, portadores de TDAH que já haviam sido diagnosticados há mais tempo e que, portanto, já estavam em tratamento relataram escores mais altos de qualidade de vida se comparados aos portadores que foram diagnosticados durante o estudo, sugerindo que o primeiro grupo possa ter se beneficiado de terapêutica específica. Esses achados demonstram que avaliações de qualidade de vida especifi- 
camente direcionadas ao TDAH podem trazer benefícios na avaliação clínica direcionando a prática terapêutica.

\section{Validação semântica em língua portuguesa e análise da confiabilidade e validade de constructo do AAQoL}

Atualmente, reconhece-se a necessidade de não apenas traduzir lingüisticamente, mas de adaptar culturalmente, de modo a manter a validade de conteúdo em nível conceitual (Guillemin et al., 1993; Beaton et al., 2000).

A validação semântica em língua portuguesa e a análise de confiabilidade e validade de constructo do AAQoL estão sendo conduzidas pelo GEDA - IPUB/UFRJ com autorização do autor da versão original. O estudo foi dividido nas seguintes fases:

1) Tradução do original em inglês e validação semântica em língua portuguesa do AAQoL;

2) Avaliação da validade do constructo;

3) Estudo de confiabilidade do instrumento, incluindo: consistência interna e estabilidade no tempo (teste-reteste).

Os indivíduos adultos serão avaliados por entrevista semi-estruturada K-SADS-PL adaptada para adultos (para diagnóstico de TDAH segundo critérios da DSM-IV), da entrevista semi-estruturada MINI-PLUS (para diagnóstico de comorbidades), sendo ainda fornecidos os instrumentos ASRS-18, SF-36 e AAQoL.

A fase de validação irá comparar os escores do AAQoL referentes ao impacto do TDAH na qualidade de vida aos escores de qualidade de vida SF-36 e pontuação (gravidade) na ASRS. Nessa fase, serão avaliados pacientes portadores de TDAH, adultos com sintomas de TDAH, porém em número insuficiente para o diagnóstico (abaixo do ponto de corte estabelecido pelo DSM-IV) e controles. Cinco hipóteses serão testadas:

a) Escores maiores no AAQoL se associam a escores menores na ASRS (quanto maiores os sintomas de TDAH, menor a qualidade de vida);

b) Escores no AAQoL são maiores para os controles em comparação aos portadores de TDAH;

c) Escores no AAQoL são maiores para portadores de TDAH do que para indivíduos com sintomas de TDAH que procuram tratamento, porém sem diagnóstico clínico (casos subsindrômicos);

d) Escores no AAQoL serão menores na presença de comorbidade ao TDAH;

e) Identificar a existência de correlação entre os escores do AAQoL e da SF-36.

\section{CONCLUSÕES}

Tendo em vista o significativo comprometimento associado à presença do TDAH na vida adulta, evidencia-se a necessi- dade de investigar o impacto dos sintomas na qualidade de vida dos portadores do transtorno. Esse aspecto pode ser particularmente importante para a investigação do critério C do sistema DSM (demonstração da existência de comprometimento funcional em pelo menos duas áreas).

O desenvolvimento de instrumento específico (AAQoL) a partir da sistematização de um conjunto de informações sobre sintomas e sobre o impacto da doença colhido com portadores, especialistas em TDAH e na literatura especializada possibilitou a criação de um modelo conceitual de investigação de qualidade de vida do indivíduo portador de TDAH. A validação semântica em língua portuguesa e a análise de confiabilidade e validade de constructo do AAQoL permitirão brevemente sua utilização em nosso meio.

Potenciais conflitos de interesse: $O$ GEDA - UFRJ recebe suporte de pesquisa do Laboratório Janssen-Cilag.

\section{REFERÊNCIAS}

Adler LA, Sutton VK, Moore RJ, Dietrich AP, Reimherr FW, Sangal B et al. Quality of life assessment in adult patients with attention-deficit/hyperactivity disorder treated with atomoxetine. J Clin Psychopharmacol, 26:648-52, 2006.

Barkley RA, Fischer M, Smallish L, Fletcher K. The persistence of attention-deficit/hyperactivity disorder into young adulthood as a function of reporting source and definition of disorder. J Abnorm Psychol, 111(2):279-89, 2002.

Beaton DE, Bombardier C, Guillemin F, Ferraz MB. Guidelines for the process of cross-cultural adaptation of self-reported measures. Spine, 25:3186-91, 2000.

Biederman J, Mick E, Faraone SV. Age-dependent decline of symptoms of attention deficit hyperactivity disorder: impact of remission definition and symptom type. Am J Psychiatry, 157(5):816-8, 2000.

Brod M, Johnston J, Able S, Swindle R. Validation of the adult attention-deficit/hyperactivity disorder quality-of-life scale (AAQoL): A disease-specific quality-of-life measure. Quality of Life Research, 15:117-29, 2006.

Grevet EH, Bau CH, Salgado CA, Ficher A, Victor MM, Garcia C et al. Concordância entre observadores para o diagnóstico em adultos do transtorno de déficit de atenção/hiperatividade e transtorno de oposição desafiante utilizando o K-SADS-E. Arq Neuropsiquiatr, 63(2):307-10, 2005.

Guillemin F, Bombardier C, Beaton D. Cross-cultural adaptation of health-related quality of life measures: literature review and proposed guidelines. J Clin Epidemiol, 46:1417-32, 1993.

Kessler RC, Adler L, Ames M, Demler 0, Faraone S, Hiripi E et al. The World Health Organization Adult ADHD Self-Report Scale (ASRS): a short screening scale for use in the general population. Psychol Med, 35(2):245-56, 2005

Kessler RC, Adler L, Barkley R, Biederman J, Conners CK, Demler 0 et al. The prevalence and correlates of adult ADHD in the United States: results from the National Comorbidity Survey Replication. Am J Psychiatry, 163: 716-23, 2006.

Kooij JJ, Buitelaar, Van Der Ooord DJ, Furer JW, Rijnders CA, Hodiamont PP. Internal and external validity of attention-deficit hyperactivity disorder in a population-based sample of adults. Psychol Med, 35(6):817-27, 2005.

Landgraf JM. Monitoring Quality of Life in Adults with ADHD: Reliability and validity of a new measure. J Att Dis, in press.

Mattos $P$, Rohde LA. Letter to the editor. J of Att Dis, in press.

Mattos P, Segenreich D, Saboya S, Louzã M, Dias G, Romano M. Adaptação transcultural para o português da escala Adult Self-Report Scale para avaliação do transtorno de déficit de atenção/hiperatividade (TDAH) em adultos. Rev Psiq Clin, 33(4):188-94, 2006.

Ware JE, Kosinski M, Dewey JE. How to score version 2 of the SF-36 Healthy Survey. Lincoln, Rl: QualityMetric, Inc.; 2000. 